\title{
INADEQUACY OF ORDINARY HOMOLOGY THEORY ${ }^{1}$
}

\author{
T. Y. LIN
}

\begin{abstract}
Counterexamples to the homology version of Peterson's theorem are constructed. Namely, maps are exhibited which theoretically cannot be detected by any primary or higher order homology operations.
\end{abstract}

1. Introduction. In homotopy theory, cohomology operations are fundamental tools. The reason for this is given, at least stably, by Peterson's theorem: Every stable map is detectable by some primary or higher order cohomology operations [6]. Recently F. Adams pointed out that, in the generalized theories, homology seems more preferable than cohomology [1]. Accordingly, it is natural to ask "Does the analogue of Peterson's theorem for homology hold true?" Unfortunately we found some maps which are theoretically undetectable by any primary or higher order homology operations. This means that, for homotopy purposes the ordinary homology theory seems inadequate. ${ }^{2}$

The author would like to thank the referee for his valuable suggestions which brought the paper into the present form.

\section{An example.}

2.1 EXAMPLE. Let $Q$ be the additive group of the rational numbers, and $L$ be the Moore space of type $(Q, n), n$ large. Let $\left\{g: S^{n} \rightarrow L, \alpha \in J\right\}$ be a set of generators of $\pi_{n}(L)$. Write $N=\mathrm{V}_{J} S^{n}, h=\mathrm{V}_{J} g_{\alpha}$; then $h: N \rightarrow L$ is a map such that $h \mid S^{n}=g_{\alpha}$. Let $C_{h}$ be the mapping cone of $h$. Then the natural inclusion $j: L \rightarrow C_{h}$ is a map undetectable by homology operations.

Proof. (i) Note that $H_{*}(L ; Z)=H_{n}(L ; Z)=Q$ is a torsion free group, and $H_{*}(N ; Z)=H_{n}(N ; Z)=F$ is a free abelian group (since $N$ is a wedge

Presented to the Society, June 25, 1970 under the title Adequacy and inadequacy of ordinary homology theory; received by the editors November 20,1970 and, in revised form, April 27, 1971.

AMS 1970 subject classifications. Primary 55G20.

Key words and phrases. Homology operation, cohomology operation, Moore space, stable homotopy ring, flat module, Hurewicz theorem.

${ }^{1}$ The preparation of this paper was sponsored in part by the National Science Foundation Grant GP-21062.

${ }^{2} \mathrm{~F}$. Adams told the author that this map can be detected by elements in $\operatorname{Ext}_{A}\left(H_{*}(S L), H_{*}\left(C_{h}\right)\right)$, where $A$ is the Steenrod algebra of integral coefficient.

(C) American Mathematical Society 1972 
of $n$-spheres). Thus, by the universal coefficient theorem, for any group $G$,

$$
\begin{aligned}
H_{i}(L ; G) & =Q \otimes G, & & i=n, \\
& =0, & & i \neq n, \\
H_{i}(N ; G) & =F \otimes G, & & i=n, \\
& =0, & & i \neq n .
\end{aligned}
$$

From construction of $h$, the map $\pi_{n}(h): \pi_{n}(N) \rightarrow \pi_{n}(L)$ is epimorphic, which, by Hurewicz theorem, is equal to $H_{n}(h ; Z): F \rightarrow Q$. Thus, by the right exactness of tensor product, the map

$$
H_{n}(h ; G)=H_{n}(h ; Z) \otimes G: F \otimes G \rightarrow Q \otimes G
$$

is also epimorphic.

Therefore, from the exactness of the homology Puppe sequence of $h$ and (1), we conclude that

is always zero.

$$
H_{*}(j ; G): H_{*}(L ; G) \rightarrow H_{*}\left(C_{h} ; G\right)
$$

(ii) Since the mapping cone $C_{j}$ is homotopy equivalent to suspension of $C_{h}, S C_{h}$, it is sufficient to show that $C_{h}$ is homotopy equivalent to a wedge of spheres. To do this, note that the homology Puppe sequence of $h$ reduces to a short exact sequence

Thus

$$
0 \rightarrow H_{n+1}\left(C_{h} ; Z\right) \rightarrow F \rightarrow Q \rightarrow 0 .
$$

$$
\begin{aligned}
H_{i}\left(C_{h} ; Z\right) & =\text { a free group, } & & i=n+1, \\
& =0, & & i \neq n+1 .
\end{aligned}
$$

Then, by Hurewicz theorem, we can show that $C_{h}$ is homotopy equivalent to a wedge of spheres (e.g. see [4, p. 134, Corollary 4.3]).

(iii) $j$ can not be null-homotopic since otherwise the Puppe sequence of $h$ would reduce to $N \simeq S C_{h} \vee L$, whence $Q=H_{n}(L ; Z)$ is a direct summand of $F=H_{n}(N ; Z)$ which is absurd.

3. More examples. Let $L$ be a connected spectrum such that the stable homotopy module $\pi_{*}(L)$ is a flat module over the stable homotopy ring $\pi_{*}$. Let $\left\{g_{\alpha}: S^{n_{\alpha} \rightarrow L}\right\}$ be a set of generators of $\pi_{*}(L)$ as $\pi_{*}$-module. Write $N=\bigvee S^{n}, h=\bigvee g_{\alpha}$, then the natural inclusion $j: L \rightarrow C_{h}$ is a map undetectable by homology operations.

The proof is similar to $\S 2$ except in (ii) we have to use [3]. The detail is omitted.

4. Remarks. 1. The reasons for existence of such a map, perhaps, can be explained by the following phenomena: (i) there are flat stable homotopy 
modules which are not projective (and hence free), or (ii) there are Moore spaces for homology but not co-Moore spaces for cohomology [5]. These two reasons are, however, essentially the same; they are bridged together in [3].

2. This phenomena can only occur in infinite complexes, such as $M(Q, n)$, since the flat stable homotopy modules of finite complexes are always projective (and hence free) [2].

\section{REFERENCES}

1. F. Adams, Lectures on generalised cohomology theory, Lectures Notes in Math., no. 99, Springer-Verlag, New York, 1968, pp. 1-138.

2. T. Lin, Homological algebra of stable homotopy ring $\pi_{*}$ of spheres, Pacific J. Math. 38 (1971).

3. - Homological dimension of $\pi_{*}$-modules and their geometric characterizations, Trans. Amer. Math. Soc. (to appear).

4. P. Freyd, Stable homotopy, Proc. Conference Categorical Algebra (La Jolla, Calif. 1965), Springer, New York, 1966, pp. 121-172. MR 35 \#2280.

5. D. M. Kan and G. W. Whitehead, On the realizability of singular cohomology groups, Proc. Amer. Math. Soc. 12 (1961), 24-25. MR 23 \#A647.

6. F. P. Peterson, Functional cohomology operations, Trans. Amer. Math. Soc. 86 (1957), 197-211. MR 21 \#4417.

Division of Mathematical Sciences, Purdue University, Lafayette, Indiana 47907 